\title{
Rediscovery of the Sixth Star Cluster in the Fornax Dwarf Spheroidal Galaxy
}

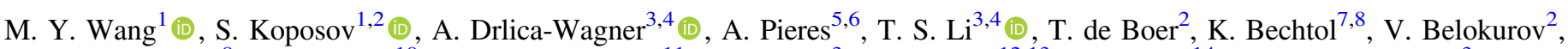

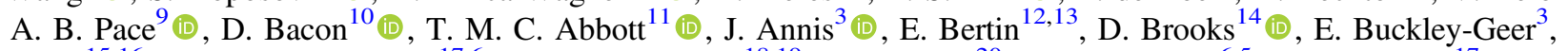
D. L. Burke ${ }^{15,16}$ (1) A. Carnero Rosell ${ }^{17,6}$, M. Carrasco Kind ${ }^{18,19}$, J. Carretero ${ }^{20}$, L. N. da Costa ${ }^{6,5}$, J. De Vicente ${ }^{17}$, S. Desai $^{21}$, H. T. Diehl ${ }^{3}$, P. Doel ${ }^{14}$, J. Estrada ${ }^{3}$, B. Flaugher ${ }^{3}$, P. Fosalba ${ }^{22,23}$, J. Frieman ${ }^{3,4}$, J. García-Bellido $^{24}$ (1), D. W. Gerdes ${ }^{25,26}$ (1), D. Gruen ${ }^{27,15,16}$ (1) R. A. Gruendl ${ }^{18,19}$ (1), J. Gschwend ${ }^{6,5}$, G. Gutierrez ${ }^{3}$, D. L. Hollowood ${ }^{28}$ (1) K. Honscheid ${ }^{29,30}$, B. Hoyle ${ }^{31,32}$, D. J. James ${ }^{33}$ (1) S. Kent ${ }^{3,4}$, K. Kuehn ${ }^{34}$ (1), N. Kuropatkin ${ }^{3}$, M. A. G. Maia ${ }^{6,5}$, J. L. Marshall ${ }^{9}$ (1), F. Menanteau ${ }^{18,19}$,

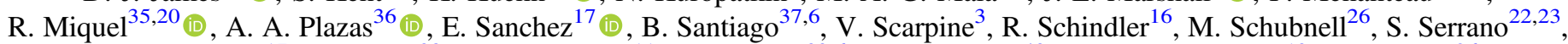

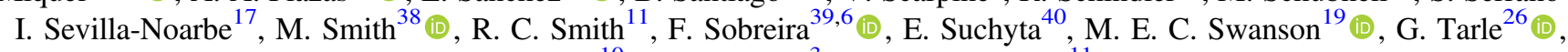
D. Thomas ${ }^{10}$, D. L. Tucker ${ }^{3}$, and A. R. Walker ${ }^{11}$ (1)

(DES Collaboration)

${ }^{1}$ Department of Physics, Carnegie Mellon University, Pittsburgh, Pennsylvania 15312, USA

2 Institute of Astronomy, University of Cambridge, Madingley Road, Cambridge CB3 OHA, UK

${ }^{3}$ Fermi National Accelerator Laboratory, P.O. Box 500, Batavia, IL 60510, USA

${ }^{4}$ Kavli Institute for Cosmological Physics, University of Chicago, Chicago, IL 60637, USA

${ }_{5}$ Observatório Nacional, Rua Gal. José Cristino 77, Rio de Janeiro, RJ-20921-400, Brazil

${ }^{6}$ Laboratório Interinstitucional de e-Astronomia-LIneA, Rua Gal. José Cristino 77, Rio de Janeiro, RJ-20921-400, Brazil

${ }^{7}$ LSST, 933 North Cherry Avenue, Tucson, AZ 85721, USA

${ }^{8}$ Physics Department, 2320 Chamberlin Hall, University of Wisconsin-Madison, 1150 University Avenue Madison, WI 53706-1390, USA

${ }^{9}$ George P. and Cynthia Woods Mitchell Institute for Fundamental Physics and Astronomy, and Department of Physics and Astronomy, Texas A\&M University, College Station, TX 77843, USA

${ }^{10}$ Institute of Cosmology and Gravitation, University of Portsmouth, Portsmouth, PO1 3FX, UK

${ }^{11}$ Cerro Tololo Inter-American Observatory, National Optical Astronomy Observatory, Casilla 603, La Serena, Chile

${ }^{12}$ CNRS, UMR 7095, Institut d'Astrophysique de Paris, F-75014, Paris, France
${ }^{13}$ Sorbonne Universités, UPMC Univ Paris 06, UMR 7095, Institut d'Astrophysique de Paris, F-75014, Paris, France

${ }^{14}$ Department of Physics \& Astronomy, University College London, Gower Street, London, WC1E 6BT, UK

${ }^{15}$ Kavli Institute for Particle Astrophysics \& Cosmology, P.O. Box 2450, Stanford University, Stanford, CA 94305, USA

${ }^{16}$ SLAC National Accelerator Laboratory, Menlo Park, CA 94025, USA

${ }^{17}$ Centro de Investigaciones Energéticas, Medioambientales y Tecnológicas (CIEMAT), Madrid, Spain

${ }^{18}$ Department of Astronomy, University of Illinois at Urbana-Champaign, 1002 W. Green Street, Urbana, IL 61801, USA

${ }^{19}$ National Center for Supercomputing Applications, 1205 West Clark Street, Urbana, IL 61801, USA
${ }^{20}$ Institut de Física d'Altes Energies (IFAE), The Barcelona Institute of Science and Technology, Campus UAB, E-08193 Bellaterra (Barcelona) Spain

${ }_{22}^{21}$ Department of Physics, IIT Hyderabad, Kandi, Telangana 502285, India

${ }^{22}$ Institut d'Estudis Espacials de Catalunya (IEEC), E-08034 Barcelona, Spain

${ }^{23}$ Institute of Space Sciences (ICE, CSIC), Campus UAB, Carrer de Can Magrans, s/n, E-08193 Barcelona, Spain

${ }^{24}$ Instituto de Fisica Teorica UAM/CSIC, Universidad Autonoma de Madrid, E-28049 Madrid, Spain

25 Department of Astronomy, University of Michigan, Ann Arbor, MI 48109, USA

${ }^{26}$ Department of Physics, University of Michigan, Ann Arbor, MI 48109, USA

${ }^{27}$ Department of Physics, Stanford University, 382 Via Pueblo Mall, Stanford, CA 94305, USA

${ }^{29}$ Santa Cruz Institute for Particle Physics, Santa Cruz, CA 95064, USA

${ }^{30}$ Department of Physics, The Ohio State University, Columbus, OH 43210, USA

${ }^{31}$ Max Planck Institute for Extraterrestrial Physics, Giessenbachstrasse, D-85748 Garching, Germany

${ }^{32}$ Universitäts-Sternwarte, Fakultät für Physik, Ludwig-Maximilians Universität München, Scheinerstr. 1, D-81679 München, Germany

${ }^{33}$ Harvard-Smithsonian Center for Astrophysics, Cambridge, MA 02138, USA

${ }^{34}$ Australian Astronomical Optics, Macquarie University, North Ryde, NSW 2113, Australia

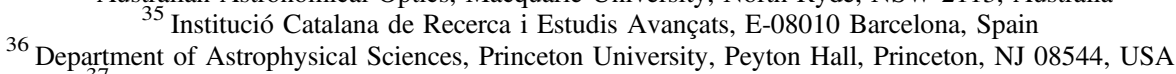

${ }^{37}$ Instituto de Física, UFRGS, Caixa Postal 15051, Porto Alegre, RS-91501-970, Brazil

${ }^{38}$ School of Physics and Astronomy, University of Southampton, Southampton, SO17 1BJ, UK

${ }^{39}$ Instituto de Física Gleb Wataghin, Universidade Estadual de Campinas, 13083-859, Campinas, SP, Brazil

${ }^{40}$ Computer Science and Mathematics Division, Oak Ridge National Laboratory, Oak Ridge, TN 37831, USA

Received 2019 February 11; revised 2019 March 28; accepted 2019 March 31; published 2019 April 16

\begin{abstract}
Since first noticed by Shapley in 1939, a faint object coincident with the Fornax dwarf spheroidal has long been discussed as a possible sixth globular cluster (GC) system. However, debate has continued over whether this overdensity is a statistical artifact or a blended galaxy group. In this Letter we demonstrate, using deep DECam imaging data, that this object is well resolved into stars and is a bona fide star cluster. The stellar overdensity of this cluster is statistically significant at the level of $\sim 6-6.7 \sigma$ in several different photometric catalogs including Gaia. Therefore, it is highly unlikely to be caused by random fluctuation. We show that Fornax 6 is a star cluster with a peculiarly low surface brightness and irregular shape, which may indicate a strong tidal influence from its host galaxy. The Hess diagram of Fornax 6 is largely consistent with that of Fornax field stars, but it appears to be
\end{abstract}


slightly bluer. However, it is still likely more metal-rich than most of the GCs in the system. Faint clusters like Fornax 6 that orbit and potentially get disrupted in the centers of dwarf galaxies can prove crucial for constraining the dark matter distribution in Milky Way satellites.

Key words: galaxies: star clusters: general

Supporting material: FITS file

\section{Introduction}

The first globular cluster (GC) around the Milky Way (MW) was discovered by Jonathan Ihle in 1655 . Since then, the sample size of MW GCs has grown to more than 100 (Harris 1996, 2010). Other than those clusters that are solely satellites of the MW, there is a particular noteworthy group that orbit in the MW dwarf satellite galaxies. All three of the most luminous MW satellites, the Large and Small Magellanic Cloud, and the Sagittarius dwarf spheroidal (dSph) galaxy have large populations of well-studied GCs (Mackey \& Gilmore 2003a, 2003b, 2003c; McLaughlin \& van der Marel 2005). The Fornax dSph galaxy, which is the fourth most luminous MW satellite, is known to possess five GCs. Up until recently, when a peculiar faint cluster was found in an ultra-faint system, Eridanus II (Koposov et al. 2015; Crnojević et al. 2016), those were the only four known MW satellites with GCs.

Among those systems, clusters in the Fornax dSph are particularly interesting. The fact that Fornax has at least five of them implies a high ratio of GC mass versus halo mass although the relation exhibits large scatter at low mass (Harris et al. 2017; Forbes et al. 2018). The existence of this handful of GCs also presents a puzzle for understanding their survival over a Hubble time (Goerdt et al. 2006; Peñarrubia et al. 2009; Cole et al. 2012; Boldrini et al. 2019). The mechanism of dynamical friction can cause those clusters to sink to the Fornax center and/or be disrupted. This so-called "Fornax timing problem" provides powerful constraints on the inner dark matter distribution of the Fornax dSph.

Despite Fornax's already high number of GCs, there is a long and often forgotten debate in the literature about a possible sixth GC, named Fornax 6. First mentioned by Shapley in 1939 (Shapley 1939), it was later observed in greater detail by a few groups in the 80's and 90's (Verner et al. 1981; Demers et al. 1994; Stetson et al. 1998). However, it was then thought to be a mixture of distant galaxies and stars. In this Letter we utilize several data sets to investigate the properties of Fornax 6 and demonstrate that it is a diffuse but bona fide star cluster that is likely undergoing tidal disruption.

\section{Data}

Fornax 6 is a compact stellar overdensity in the projected central area of the Fornax dSph (see Figure 2). It was noticed by the authors during visual inspections of the DES Y3 coadded images. Because of crowding issues at the center of Fornax dSph (Wang et al. 2019), most of the objects in the overdensity are missing in the internal DES Y3 and the public DES DR1 source catalogs (DES Collaboration et al. 2018; Morganson et al. 2018). Although the rediscovery of the overdensity was done using DES imaging, we searched for best quality publicly available data that has covered the same area. We found that the area around Fornax 6 was observed in $r$ and $i$ bands during exceptional seeing conditions of $0 . " 6-0$ ". 7 by a DECam program 2016B-0244 (PI: B. Tucker) that is available in the NOAO Science Archive. We therefore use the photometrically and astrometrically calibrated catalog generated by running the DECam community pipeline (Valdes et al. 2014) on this imaging data for the remainder of the paper. We stack $\sim 6 r$ - and $i$-band images using SWARP software (Bertin 2010) and calibrate the photometry by computing zero-points from the cross-match in the DES DR1 catalog. To alleviate crowding issues we ran DAOPHOT (Stetson 1987; Bradley et al. 2018) software on a region of $6^{\prime} \times 6^{\prime}$ around the overdensity.

We also utilize Gaia Data Release 2 (DR2) data (Gaia Collaboration et al. 2018) for additional photometry and proper motion (PM) information. In order to reject extended sources, we adopt the following magnitude-dependent cut on the astrometric_excess_noise (AEN) parameter (Koposov et al. 2017):

$$
\log (\mathrm{AEN})<1.5+0.3(\mathrm{G}-18) .
$$

Additionally we use the data from the VST ATLAS survey (Shanks et al. 2015) that was reprocessed and calibrated by Koposov et al. (2014), and spectroscopic data from Walker et al. (2009).

\section{Objects in Fornax 6}

In Figure 1 we show the false color gri coadded image of $\sim 20^{\prime} \times 20^{\prime}$ field around the center area of the Fornax dSph from DES. The position of Fornax 6 (marked by an orange arrow) is $\sim 7^{\prime}$ north from Fornax 4 (another Fornax GC, marked by a blue arrow) as described in the literature (e.g., Shapley 1939). It is the second closest cluster to the Fornax center in terms of projected distance $(\sim 0.27 \mathrm{kpc})$ other than Fornax $4(\sim 0.15 \mathrm{kpc})$. The Fornax dSph optical center location adopted here is from recent photometry studies (e.g., Bate et al. 2015; Wang et al. 2019).

However, its luminosity and morphology seem peculiar among the known Fornax GCs, as it appears visually much fainter than other clusters. Also, while GCs are typically spherical, Fornax 6 has a nonnegligible ellipticity (e.g., see Figures 1 and 2). In the left panel of Figure 2 we show the false color gri DES coadded image of $2^{\prime} \times 2^{\prime}$ field centered on Fornax 6. Several literature sources (e.g., Verner et al. 1981; Stetson et al. 1998) have considered Fornax 6 to be a mixture of stars and galaxies. However, most of the objects discussed in Stetson et al. (1998) that were thought to be nonstellar, are clearly multiple closely located stars in the DES coadded image. Although a few of the objects are still likely distant galaxies, their contribution is likely not significant.

In the right four panels of Figure 2 we show the density distributions of sources from DES Y3, VST, Gaia DR2, and the DAOPHOT catalogs constructed from the DECam images. We note that barring Gaia data we do not apply any star/ galaxy separation criteria as most of the sources are expected to be stars. Fornax 6 appears as a prominent overdensity in VST, Gaia, and DECam data, with the exception of DES Y3, where 


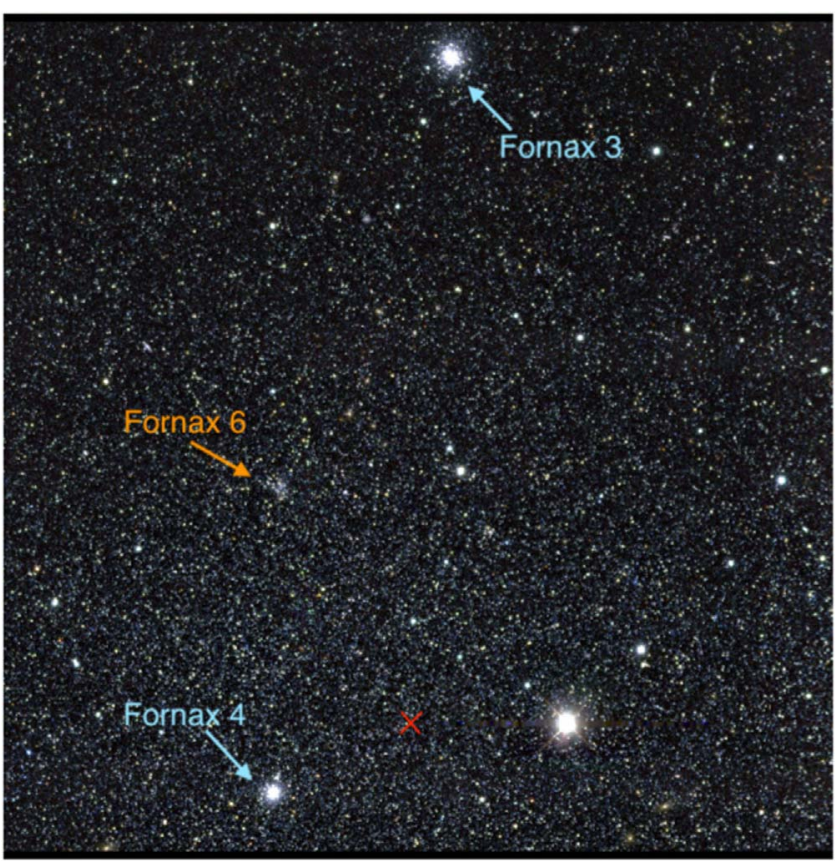

Figure 1. False color gri coadded image of $\sim 20^{\prime} \times 20^{\prime}$ field around the center area of the Fornax dSph galaxy from DES. The locations of several Fornax GCs including Fornax 6 are marked by blue and orange arrows. The red cross marks the center of the Fornax dSph galaxy from Wang et al. (2019).

it is underdense, in part due to a known limitation of catalogs generated by SExtractor (Bertin et al. 2002) in a dense star field.

We also remark that in several studies Fornax 6 has been discussed as a statistical artifact caused by random clustering (e.g., Demers et al. 1995). To validate the significance of the overdensity, we randomly draw 10,000 subsamples from the DAOPHOT and the Gaia DR2 catalog within a search radius of 13 ". 2 in a $6^{\prime} \times 6^{\prime}$ field excluding area within 33.6 arcsec (two times the project half-light radius $r_{h}$ derived in Section 4.2) centered on Fornax 6 . The search radius of 13 !" 2 is chosen to maximize the significance. The average and the standard deviation of the counts from the DAOPHOT (Gaia) catalog sampling is $48.9 \pm 6.6(4.0 \pm 2.0)$. The Fornax 6 star number count within 13 !' 2 is 96 (16), which is $6.7(6.0) \sigma$ above the background assuming a Poisson distribution. We note that only 1 out of 10,000 random subsamples has significance above $4 \sigma$ (which is $4.2 \sigma$ ) in the DAOPHOT catalog. For Gaia we test eight additional fields at the same distance from the Fornax dSph center. Five out of the eight fields have 1 out of 10,000 samples with significance above $4 \sigma$ (the highest one is $4.9 \sigma$ ). Therefore, we conclude that Fornax 6 is highly unlikely to be a random statistical fluctuation.

\section{Properties of Fornax 6}

\subsection{Hess Diagram}

In the middle panel of Figure 3 we show the extinctioncorrected color-magnitude diagram (CMD) of Fornax 6 (red points) within 16 ". 8 (the size of $r_{h}$ ) of the best-fit centroid from the DECam DAOPHOT catalog. The CMD of the Fornax dSph, drawn from a $5^{\prime} \times 5^{\prime}$ field centered on Fornax 6, while excluding the region within $2 r_{h}$ of the cluster center, is shown with black dots. In general, the CMD of Fornax 6 is largely consistent with the Fornax dSph CMD.
The background-subtracted star count histograms (with respect to the Fornax dSph field stars) of Fornax 6, which is shown in the right most panel in Figure 3, peaks at similar magnitude $(i \sim 20.5)$ as the Fornax dSph. However, this peak in the Fornax dSph is dominated by red-clump (RC) stars while in Fornax 6 it is likely dominated by the red horizontal branch (RHB) stars. Nevertheless, the similar magnitude locations of these peaks indicate that they are at comparable distances. On the top panel of Figure 3, we show the background-subtracted star counts as a function of $r-i$ color between $20.3<i<21.0$, which covers RC and horizontal branch (HB) stars. The star counts of Fornax 6 peak at slightly bluer color than Fornax $\mathrm{dSph}$. In the left panel of Figure 3 we show the backgroundsubtracted CMD of Fornax 6 colored by their significance. The subtracted CMD also shows that the red giant branch stars are slightly shifted to the bluer end with additional significant features at $\sim 4 \sigma$ at $r-i=0.0$ and $i=20.4$, likely caused by the RHB stars. This indicates that Fornax 6 may have a more metal-poor population than the inner Fornax field stars (with mean $[\mathrm{Fe} / \mathrm{H}] \sim-0.9$, Battaglia et al. 2006). However, Fornax 6 is likely to have similar metallicity as Fornax 4 (with $[\mathrm{Fe} / \mathrm{H}] \sim-1.5)$, which also presents a prominent population of RHB stars and lacks a significant blue HB population (de Boer \& Fraser 2016).

\subsection{Structural Properties and Luminosity}

We perform a Plummer model fit to the Fornax 6 stellar distribution using the 2D unbinned likelihood described in Martin et al. (2008). We include in the fits only stars with $r<22$, but we note that the fitting results might be systematically off with underestimated uncertainties due to a lack of completeness corrections. We find that Fornax 6' s stellar density profile is well-described by a Plummer profile (see Figure 4 ) with $r_{h}=16$ ". $8 \pm 2$ ". 0 , and it has high ellipticity $(\epsilon=0.41 \pm 0.10)$.

We use the posterior distributions of the structural parameters to estimate the total number of stars in the cluster. Then, the number of stars is converted into the total luminosity assuming the luminosity function is well-described by a PARSEC isochrone model (Bressan et al. 2012) with age $=10 \mathrm{Gyr}$ and $[\mathrm{Fe} / \mathrm{H}]=-1.5$ and the Chabrier initial mass function (Chabrier 2003). Assuming a distance of $147 \mathrm{kpc}$ (the distance of Fornax dSph, McConnachie 2012), the estimated absolute magnitude of Fornax 6 in $V$ band is $M_{V}=-4.8 \pm 0.4$, which is fainter than other Fornax GCs that range from $M_{V}=-8.2$ to -5.2 (Webbink 1985). A summary of Fornax 6 properties is provided in Table 1.

\subsection{Radial and PM Velocity}

Here we use Gaia to test the hypothesis of Fornax 6 belonging to the Fornax dSph. However, it is impossible to determine the PM of the whole system precisely given that the PM uncertainties

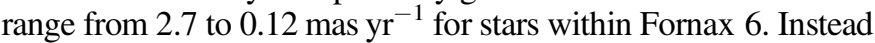
we examine how many possible members are bound. We select stars within $2 r_{h}$ of the cluster center and require that they have small parallaxes with respect to their uncertainties of $\varpi<2 \sigma_{\varpi}$. We also require their PM to be within $3 \sigma$ from the estimated escape speed (calculated using a Navarro-Frenk-White (NFW) potential) at the distance of the object with respect to the galaxy PM $\left(\mu_{\alpha^{*}}=0.375, \mu_{\delta}=-0.401\right.$ mas yr $^{-1}$, Fritz et al. 2018). In addition, we apply a CMD mask to select targets lying within $\pm 0.2 \mathrm{mag}$ to the isochrone model mentioned in 

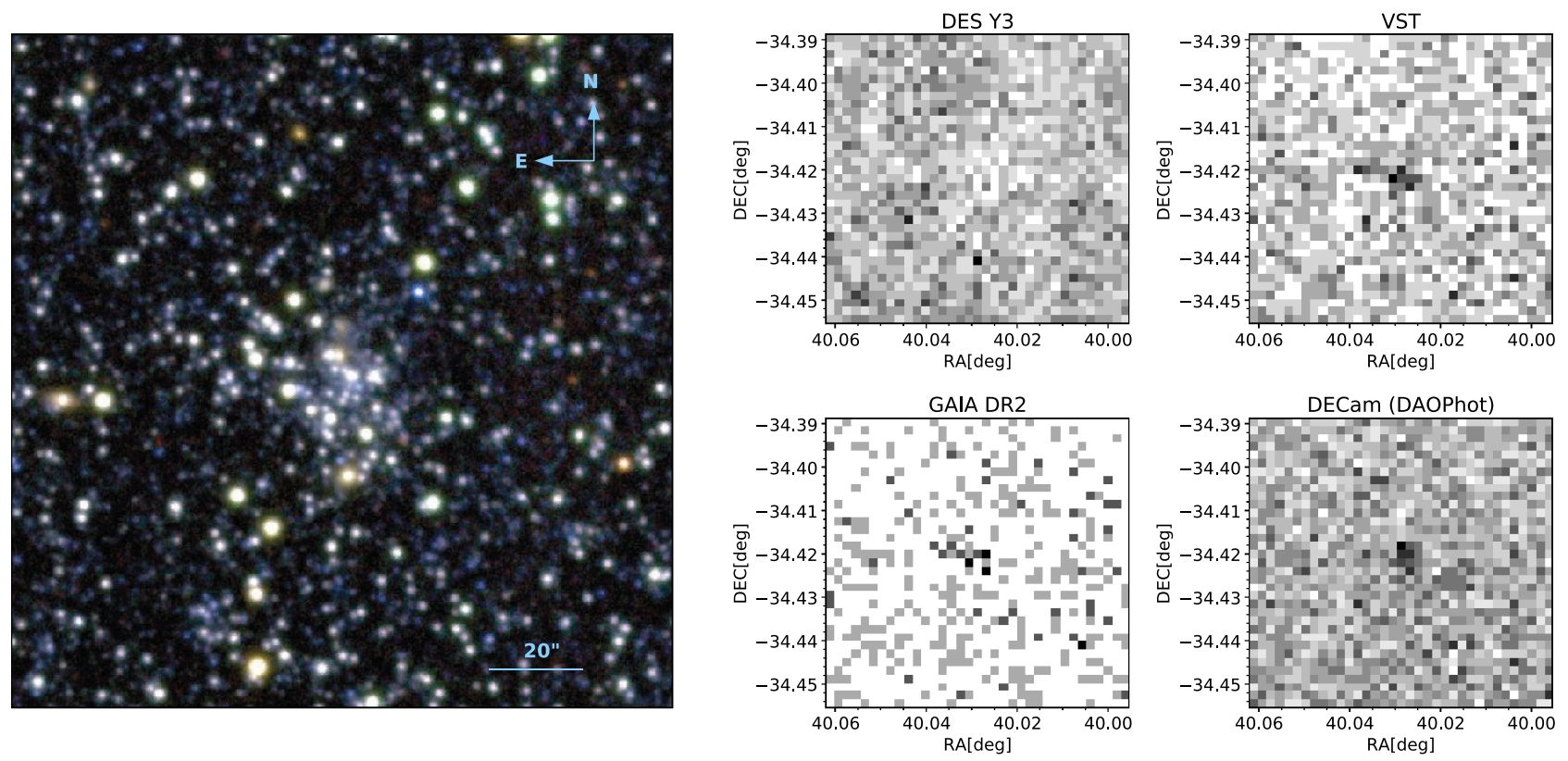

Figure 2. Left panel: false color gri coadded image of $2^{\prime} \times 2^{\prime}$ field centered on Fornax 6 from DES. Right four panels: density distribution of detected sources around Fornax 6 in a $4^{\prime} \times 4^{\prime}$ field. From left to right, top to bottom, we show data from internal DES Y3, VST ATLAS, Gaia DR2, and our DECam DAOPHOT catalogs.
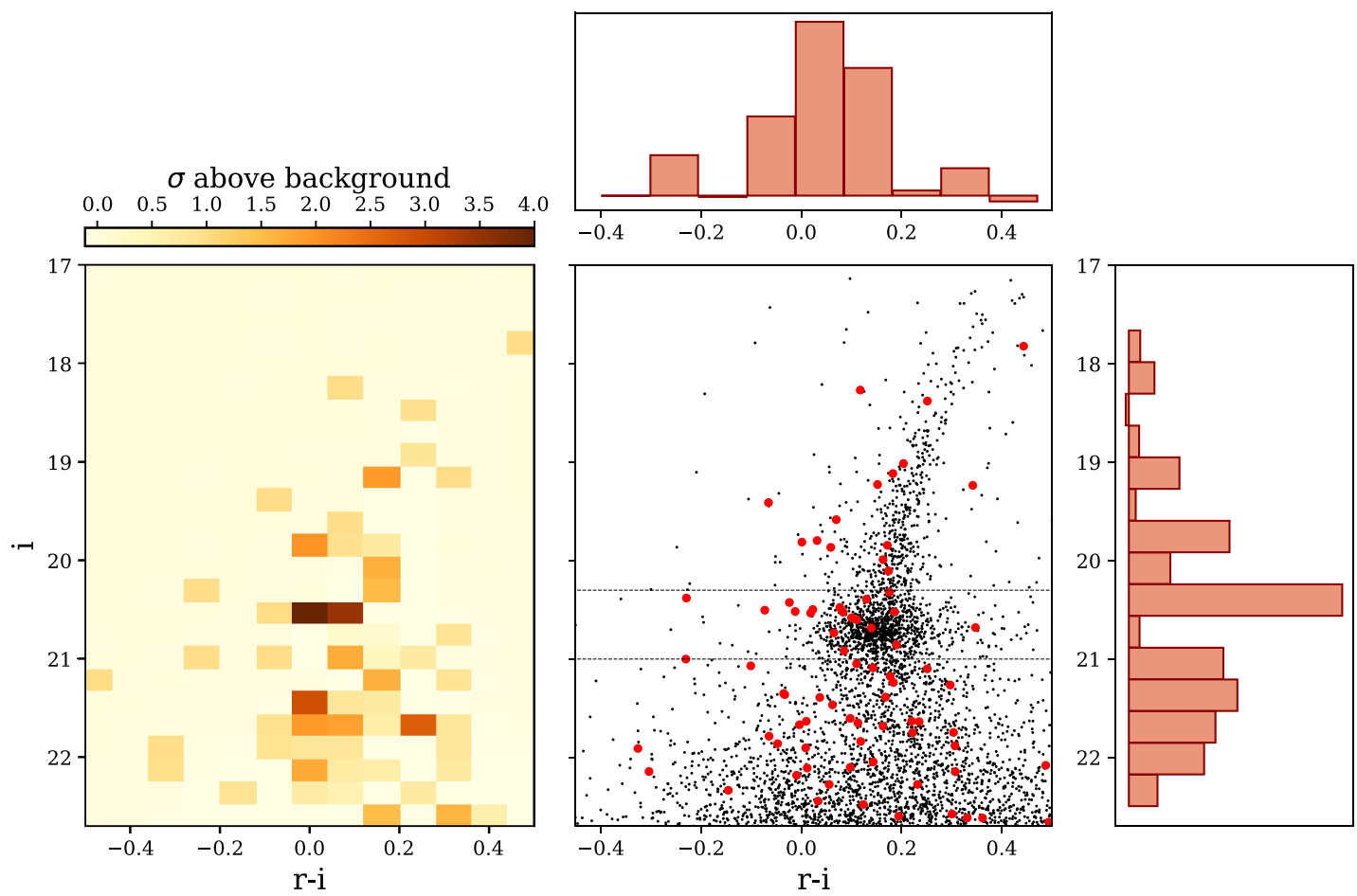

Figure 3. Left panel: background-subtracted (with respect to Fornax dSph field stars) CMD of Fornax 6 colored by Poisson significance. Top panel: backgroundsubtracted histograms of star counts as a function of $r-i$ between $20.3<i<21.0$ for Fornax 6. Middle panel: comparison of Fornax 6 CMD within $r_{h}=16^{\prime \prime} 8$ (red points) with Fornax dSph CMD (black points). Right panel: background-subtracted histograms of star counts as a function of $i$ magnitude for Fornax 6 . The positions and magnitudes (in $r$ and $i$ ) of the resulting catalog are available in FITS format as data behind the figure.

Section 4.2. We find that 18 out of $32(56 \%)$ stars lying within $2 r_{h}$ of Fornax 6 pass these criteria, while about 8 stars $(\sim 25 \%)$ are expected from Fornax dSph field star contamination. Therefore a significant fraction of Fornax 6 stars has PM consistent with being bound to the Fornax dSph.

There is a small sample of five stars within $2.2 r_{h}$ with spectroscopic measurements from Walker et al. (2009). However, only four of them have PM consistent with being bound to the Fornax dSph. The derived mean radial velocity (RV) and velocity dispersion of these four stars are $53.9_{-1.1}^{+1.2} \mathrm{~km} \mathrm{~s}^{-1}$ and $2.1_{-0.8}^{+1.7} \mathrm{~km} \mathrm{~s}^{-1}$. Its RV is very similar to the RV of the Fornax $\mathrm{dSph}$, which is $53.3 \mathrm{~km} \mathrm{~s}^{-1}$ (Walker et al. 2009). We note that $\sim 30 \%$ of stars in this sample are expected to be background stars belonging to Fornax dSph. However, if we randomly draw four 


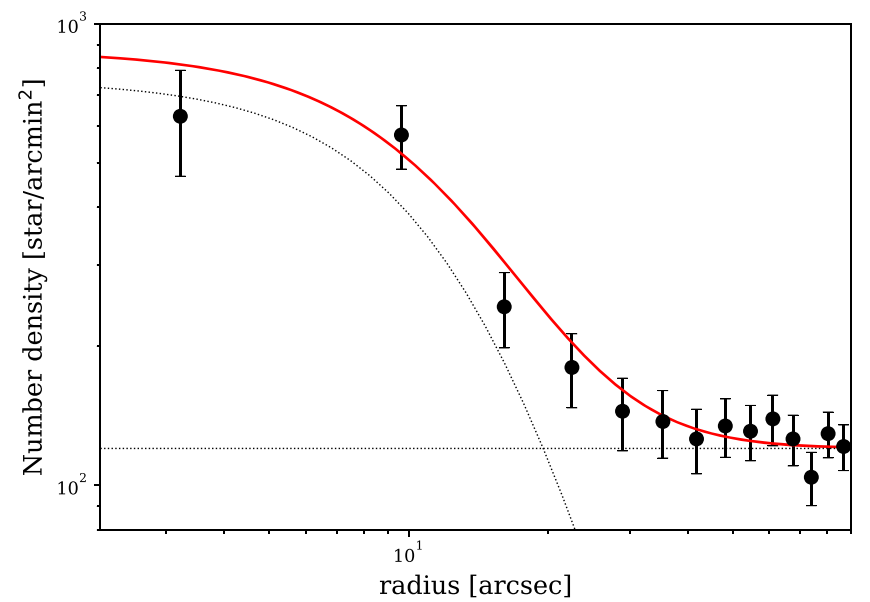

Figure 4. One-dimensional density profile of Fornax 6. The black dashed lines show the fitted Plummer and background model separately, and the red line shows their combination. Black points show the binned DAOPHOT data.

Table 1

Properties of Fornax 6

\begin{tabular}{lc}
\hline \hline Parameter & \\
\hline R.A.(J2000) & $2^{\mathrm{h}} 40^{\mathrm{m}} 6^{\mathrm{s}} \cdot 9$ \\
Decl.(J2000) & $-34^{\circ} 25^{\prime} 19^{\prime \prime} \cdot 2$ \\
$\epsilon$ & $0.41 \pm 0.10$ \\
P.A. (deg) & $13.1_{-7.3}^{+10.4}$ \\
$r_{h}(\operatorname{arcsec})$ & $16.8 \pm 2.0$ \\
$r_{h}(\mathrm{pc})$ & $11.3 \pm 1.4$ \\
$M_{V}(\mathrm{mag})$ & $-4.8 \pm 0.4$ \\
\hline
\end{tabular}

stars out of the velocity distribution of Fornax dSph, which has a velocity dispersion of $11.7 \mathrm{~km} \mathrm{~s}^{-1 * *}$ (Walker et al. 2009; McConnachie 2012), the probability of measuring dispersion less than $3.8 \mathrm{~km} \mathrm{~s}^{-1}$ (within $1 \sigma$ upper bound of our estimation) is only $\sim 6.5 \%$. Thus, under the assumption that some of the measured signals indeed correspond to Fornax 6, it is indicated that this cluster has low velocity dispersion and an RV that is similar to the Fornax dSph itself.

\section{Implications for Fornax dSph Dark Matter Distribution}

The tidal evolution of a diffuse GC like Fornax 6 may provide important clues for understanding Fornax's inner dark matter profile. For example, Peñarrubia et al. (2009) argues that surviving low-mass GCs like Fornax 1 should mostly populate the outskirts of their host galaxies to avoid being disrupted by tides. Furthermore, it is expected that orbital decay due to dynamical friction experienced by a low-mass GC would be small (e.g., Binney \& Tremaine 2008). Thus Fornax 6' s small projected distance is in tension with these arguments, although its true $3 \mathrm{D}$ distance is unknown. Therefore, there are two possible scenarios: (1) The initial mass of Fornax 6 was large and comparable to Fornax 2-5, and therefore its 3D distance could be close due to orbital decay. It also implies substantial mass loss. (2) The initial mass of Fornax 6 was small, and therefore its $3 \mathrm{D}$ distance may actually not be close to the center of Fornax.

Here we estimate the influences of tides by computing the Jacobi radius $r_{J}$. The average stellar density of the cluster within $r_{h}$ is $\sim 0.7 M_{\odot} / \mathrm{pc}^{3}$. At $r=0.27 \mathrm{kpc}$ (the projected distance to Fornax center), the Fornax dSph dark matter density $\rho_{\mathrm{dm}}$ is $\sim 0.08 M_{\odot} / \mathrm{pc}^{3}$ for an NFW potential with $V_{\max } \sim$ $30 \mathrm{~km} \mathrm{~s}^{-1}$. Therefore, the $r_{J}$ for Fornax 6 is $\sim 16 \mathrm{pc}$. This is only a factor of 1.4 times the cluster half-light radius, and therefore supports the tidal disruption hypothesis. If we assume a cored profile, such as that from Walker \& Peñarrubia (2011; see their Figure 1) that suggests $\rho_{\mathrm{dm}}$ is $\sim 0.04 M_{\odot} / \mathrm{pc}^{3}$ at $r=0.27 \mathrm{kpc}$; the predicted $r_{J}$ is $\sim 21 \mathrm{pc}$ (1.9 times the halflight radius). We also remark that Fornax 6 could be strongly affected by tides in an NFW potential even if it is significantly farther away from the Fornax center, as the $r_{J}$ is smaller than $2 \times r_{h}$ for $3 \mathrm{D}$ separations up to $0.8 \mathrm{kpc}$. In contrast, in the cored profile, the $r_{J}$ stays $\sim 1.8-2.0 \times r_{h}$ anywhere within a 3D distance of $0.8 \mathrm{kpc}$, indicating limited tidal influences when the inner host potential is shallow.

Due to its small mass, this rediscovered star cluster does not add much to the total GC mass budget of the Fornax dSph, and therefore does not impact on the established GC mass-halo mass relation. However, the total GC mass budget within a galaxy may have been underestimated due to the substantial amount of tidally stripped stars buried in the galaxy's dense stellar field.

\section{Conclusion}

In this Letter we demonstrate that Fornax 6, which was historically thought to be a dubious object, is a genuine star cluster within the Fornax dSph. Using deep DES Y3 coadded images we show that a few objects within Fornax 6 that are claimed to be galaxies in the literature are actually blended images of multiple stars. The stellar overdensity caused by Fornax 6 has a significance of $6-6.7 \sigma$ in the DECam and the Gaia DR2 photometry catalogs. We show that Fornax 6's CMD is largely consistent with Fornax dSph, but slightly shifted to the bluer end.

Fornax 6' s light profile is well-fit by a Plummer model with $r_{h}=11.3 \pm 1.4 \mathrm{pc}$ and high ellipticity of $\epsilon=0.41 \pm 0.1$, with an estimated luminosity of $M_{V}=-4.8 \pm 0.4$. The highly elongated shape of Fornax 6 suggests that it is undergoing tidal disruption.

We also check the available kinematic information to assess how likely Fornax 6 is bound to the Fornax dSph. By examining stars in Gaia, we show that a high fraction of stars within Fornax 6 have PM consistent with being bound to the Fornax dSph. Four possible members of Fornax 6 with spectroscopic data also suggest RV close to the velocity of the Fornax dSph with low velocity dispersion, but this needs to be verified with more members.

Since Fornax 6 may be the only Fornax GC that shows clear signs of tidal disruption, its tidal evolution can provide a powerful probe to the Fornax dSph dark matter potential. Depending on the assumptions about the initial mass of Fornax 6 and its 3D distance to the Fornax center, the dynamical friction and tidal force it experienced can vary. Detailed $N$-body simulations will be needed to quantify these effects.

M.Y.W. acknowledges support of the McWilliams Postdoctoral Fellowship. S.K. is partially supported by National Science Foundation grant AST-1813881.

This paper has gone through internal review by the DES collaboration.

Funding for the DES Projects has been provided by the DOE and NSF(USA), MEC/MICINN/MINECO(Spain), STFC(UK), HEFCE(UK). NCSA(UIUC), KICP(U. Chicago), CCAPP(Ohio State), MIFPA(Texas A\&M), CNPQ, FAPERJ, FINEP (Brazil), 
DFG(Germany), and the Collaborating Institutions in the Dark Energy Survey.

The Collaborating Institutions are Argonne Lab, UC Santa Cruz, University of Cambridge, CIEMAT-Madrid, University of Chicago, University College London, DES-Brazil Consortium, University of Edinburgh, ETH Zürich, Fermilab, University of Illinois, ICE (IEEC-CSIC), IFAE Barcelona, Lawrence Berkeley Lab, LMU München and the associated Excellence Cluster Universe, University of Michigan, NOAO, University of Nottingham, Ohio State University, University of Pennsylvania, University of Portsmouth, SLAC National Lab, Stanford University, University of Sussex, Texas A\&M University, and the OzDES Membership Consortium.

Based in part on observations at Cerro Tololo InterAmerican Observatory, National Optical Astronomy Observatory, which is operated by the Association of Universities for Research in Astronomy (AURA) under a cooperative agreement with the National Science Foundation.

The DES Data Management System is supported by the NSF under grant No. AST-1138766 and AST-1536171. The DES participants from Spanish institutions are partially supported by MINECO under grants AYA2015-71825, ESP2015-66861, FPA2015-68048, SEV-2016-0588, SEV-2016-0597, and MDM-2015-0509, some of which include ERDF funds from the European Union. IFAE is partially funded by the CERCA program of the Generalitat de Catalunya. Research leading to these results has received funding from the European Research Council under the European Union's Seventh Framework Program (FP7/2007-2013) including ERC grant agreements 240672, 291329, and 306478. We acknowledge support from the Australian Research Council Centre of Excellence for Allsky Astrophysics (CAASTRO), through project number CE110001020, and the Brazilian Instituto Nacional de Ciênciae Tecnologia (INCT) e-Universe (CNPq grant 465376/2014-2).

This manuscript has been authored by Fermi Research Alliance, LLC under Contract No. DE-AC02-07CH1 1359 with the U.S. Department of Energy, Office of Science, Office of High Energy Physics.

\section{ORCID iDs}

M. Y. Wang (i) https://orcid.org/0000-0002-8226-6237

S. Koposov (1) https://orcid.org/0000-0003-2644-135X

A. Drlica-Wagner (i) https://orcid.org/0000-0001-8251-933X

T. S. Li (i) https://orcid.org/0000-0002-9110-6163

A. B. Pace (1) https://orcid.org/0000-0002-6021-8760

D. Bacon (1) https://orcid.org/0000-0002-2562-8537

T. M. C. Abbott (1) https://orcid.org/0000-0003-1587-3931

J. Annis (i) https://orcid.org/0000-0002-0609-3987

D. Brooks (1) https://orcid.org/0000-0002-8458-5047

D. L. Burke (1) https://orcid.org/0000-0003-1866-1950

J. García-Bellido (1) https://orcid.org/0000-0002-9370-8360

D. W. Gerdes (i) https://orcid.org/0000-0001-6942-2736

D. Gruen (1) https://orcid.org/0000-0003-3270-7644

R. A. Gruendl (1) https://orcid.org/0000-0002-4588-6517

D. L. Hollowood (1) https://orcid.org/0000-0002-9369-4157

D. J. James (1) https://orcid.org/0000-0001-5160-4486

K. Kuehn (ㄷ) https://orcid.org/0000-0003-0120-0808

J. L. Marshall @ https://orcid.org/0000-0003-0710-9474
R. Miquel (1) https://orcid.org/0000-0002-6610-4836

A. A. Plazas (ii) https://orcid.org/0000-0002-2598-0514

E. Sanchez (1) https://orcid.org/0000-0002-9646-8198

M. Smith (1) https://orcid.org/0000-0002-3321-1432

F. Sobreira (1) https://orcid.org/0000-0002-7822-0658

M. E. C. Swanson (10 https://orcid.org/0000-0002-1488-8552

G. Tarle (1) https://orcid.org/0000-0003-1704-0781

A. R. Walker (1) https://orcid.org/0000-0002-7123-8943

\section{References}

Bate, N. F., McMonigal, B., Lewis, G. F., et al. 2015, MNRAS, 453, 690 Battaglia, G., Tolstoy, E., Helmi, A., et al. 2006, A\&A, 459, 423

Bertin, E. 2010, SWarp: Resampling and Co-adding FITS Images Together, Astrophysics Source Code Library, ascl:1010.068

Bertin, E., Mellier, Y., Radovich, M., et al. 2002, in ASP Conf. Ser. 281, Astronomical Data Analysis Software and Systems XI, ed. D. A. Bohlender, D. Durand, \& T. H. Handley (San Francisco, CA: ASP), 228

Binney, J., \& Tremaine, S. 2008, Galactic Dynamics (2nd ed.; Princeton, NJ: Princeton Univ. Press)

Boldrini, P., Mohayaee, R., \& Silk, J. 2019, MNRAS, 485, 2546

Bradley, L., Sipocz, B., Robitaille, T., et al. 2018, astropy/photutils: v0.5, Zenodo, doi:10.5281/zenodo.1340699

Bressan, A., Marigo, P., Girardi, L., et al. 2012, MNRAS, 427, 127

Chabrier, G. 2003, PASP, 115, 763

Cole, D. R., Dehnen, W., Read, J. I., \& Wilkinson, M. I. 2012, MNRAS, 426, 601

Crnojević, D., Sand, D. J., Zaritsky, D., et al. 2016, ApJL, 824, L14

de Boer, T. J. L., \& Fraser, M. 2016, A\&A, 590, A35

Demers, S., Battinelli, P., Irwin, M. J., \& Kunkel, W. E. 1995, MNRAS, 274,491

Demers, S., Irwin, M. J., \& Kunkel, W. E. 1994, AJ, 108, 1648

DES Collaboration, Abdalla, F. B., Allam, S., et al. 2018, ApJS, 239, 18

Forbes, D. A., Read, J. I., Gieles, M., \& Collins, M. L. M. 2018, MNRAS, 481, 5592

Fritz, T. K., Battaglia, G., Pawlowski, M. S., et al. 2018, A\&A, 619, A103

Gaia Collaboration, Brown, A. G. A., Vallenari, A., et al. 2018, A\&A, 616, A1

Goerdt, T., Moore, B., Read, J. I., Stadel, J., \& Zemp, M. 2006, MNRAS, 368,1073

Harris, W. E. 1996, AJ, 112, 1487

Harris, W. E. 2010, arXiv:1012.3224

Harris, W. E., Blakeslee, J. P., \& Harris, G. L. H. 2017, ApJ, 836, 67

Koposov, S. E., Belokurov, V., \& Torrealba, G. 2017, MNRAS, 470, 2702

Koposov, S. E., Belokurov, V., Torrealba, G., \& Evans, N. W. 2015, ApJ, 805,130

Koposov, S. E., Irwin, M., Belokurov, V., et al. 2014, MNRAS, 442, L85

Mackey, A. D., \& Gilmore, G. F. 2003a, MNRAS, 338, 120

Mackey, A. D., \& Gilmore, G. F. 2003b, MNRAS, 338, 85

Mackey, A. D., \& Gilmore, G. F. 2003c, MNRAS, 340, 175

Martin, N. F., de Jong, J. T. A., \& Rix, H.-W. 2008, ApJ, 684, 1075

McConnachie, A. W. 2012, AJ, 144, 4

McLaughlin, D. E., \& van der Marel, R. P. 2005, ApJS, 161, 304

Morganson, E., Gruendl, R. A., Menanteau, F., et al. 2018, PASP, 130, 074501

Peñarrubia, J., Walker, M. G., \& Gilmore, G. 2009, MNRAS, 399, 1275

Shanks, T., Metcalfe, N., Chehade, B., et al. 2015, MNRAS, 451, 4238

Shapley, H. 1939, PNAS, 25, 565

Stetson, P. B. 1987, PASP, 99, 191

Stetson, P. B., Hesser, J. E., \& Smecker-Hane, T. A. 1998, PASP, 110, 533

Valdes, F., Gruendl, R. \& DES Project 2014, in ASP Conf. Ser. 485, Astronomical Data Analysis Software and Systems XXIII, ed. N. Manset \& P. Forshay (San Francisco, CA: ASP), 379

Verner, G., Demers, S., Hardy, E., \& Kunkel, W. E. 1981, AJ, 86, 357

Walker, M. G., Mateo, M., \& Olszewski, E. W. 2009, AJ, 137, 3100

Walker, M. G., \& Peñarrubia, J. 2011, ApJ, 742, 20

Wang, M.-Y., de Boer, T., Pieres, A., et al. 2019, arXiv:1809.07801

Webbink, R. F. 1985, in Proc. IAU Symp. 113, Dynamics of Star Clusters, ed. J. Goodman \& P. Hut (Dordrecht: Reidel), 541 